\title{
The Role of Structural Characteristics in Video-Game Play Motivation: A Q-Methodology Study
}

\author{
Dave Westwood, M.Sc. and Mark D. Griffiths, Ph.D.?
}

\begin{abstract}
Until recently, there has been very little naturalistic study of what gaming experiences are like, and how gaming fits into people's lives. Using a recently developed structural characteristic taxonomy of video games, this study examined the psycho-structural elements of computer games that motivate gamers to play them. Using Q-Sort methodology, 40 gamers participated in an online Q-sort task. Results identified six distinct types of gamers based on the factors generated: (a) story-driven solo gamers; (b) social gamers; (c) solo limited gamers; (d) hardcore online gamers; (e) solo control/identity gamers; and (f) casual gamers. These gaming types are discussed, and a brief evaluation of similar and unique elements of the different types of gamer is also offered. The current study shows Q-methodology to be a relevant and applicable method in the psychological research of gaming.
\end{abstract}

\section{Introduction}

$\mathbf{R}$ ESEARCH INTO ADULT GAMING BY JANSZ AND TANIs ${ }^{1}$ found that people who played the online first-person shooter game Counterstrike were motivated by different elements depending upon how long they had been playing the game for. Those gamers who had been playing the longest and describing themselves as "semi-professional" reported being more highly motivated by the elements of challenge and competition than those gamers who had played for less time. They also concluded that social interaction was the largest motivation for playing this particular online game. Kim and Ross ${ }^{2}$ studied the motivations of gamers who played sportssimulation video games. They found that, regardless of the amount of time spent playing this genre of game, gamers were motivated by seven factors: knowledge application, identification with sport, fantasy, competition, entertainment, social interaction, and diversion.

What is needed is an understanding of how the structural elements of games are viewed together in relation to each other, and then how these motivate an individual to play. A paper by Wood et al. ${ }^{3}$ identified many structural elements that are important in playing computer games. These included sound, graphics, background and setting, game duration, rate of play, advancement rate, use of humor, control options, game dynamics, winning and losing features, character development, brand assurance, and multiplayer features. In an attempt to provide some structure to gaming research, and based on the research of Wood et al., King et al. ${ }^{4}$ constructed a new structural characteristic taxonomy that offered a psychological understanding of the structures within computer games. The taxonomy comprised five main types of structural characteristic: social (features within games that either allow for communication and the construction of relationships, or allow an individual to compare attainment against others, such as leader boards); manipulation/control (features that allow the player the option to personalize control systems, as well as the ability to save progress); narrative/identity (features that incorporate the ability for personal avatar creation, story-progression features [i.e., cut scenes], and genre-specific features); reward and punishment (features such as level and character progression, and character death); and presentation (features such as the audiovisual aspects of the game, for example, graphics, soundtrack, and advertising). This study seeks to understand the psychostructural elements of computer games that motivate gamers to play them. It aims to offer an explanation of the elements that are motivationally important to gamers and to offer an understanding of why they choose to play one game over another.

\section{Method}

\section{Participants}

The participants comprised 40 gamers (38 males and 2 females) all aged between 18 and 40 years (with $90 \%$ of the sample aged below 30 years). Participants played for a mean 
average of 11.5 hours a week, ranging from 1 to 50 hours a week.

\section{Methodology}

This study utilized Q-methodology (QM). According to Cross, ${ }^{5} \mathrm{QM}$ is ideal for researching the way people construe and construct a particular phenomenon. QM requires the participant to sort "statements" into a normally distributed hierarchical pyramid. The "sorts" are then analyzed using an inverted factor analysis. These analyses look for overarching "profiles" of how people are similarly construing and constructing the area under empirical investigation. According to Watts and Stenner, ${ }^{6}$ the optimum number of participants in these types of study is between 40 and 60 (in order to maintain the subjective nature of $\mathrm{QM}$, and to allow all participants' voices to be "heard"). The most important part of QM studies is the sample statements. These must incorporate every aspect of the phenomenon being investigated. Here, initial statements were constructed from the taxonomy outlined above (2009). The initial statement list comprised 60 statements but was reduced to 56 following focus-group discussions with gamers. This was conducted so that the statements covered every aspect of gaming and so that the wording of each statement was gamer friendly. (A full set of the statements is available from the authors.)

\section{Procedure}

Participants were recruited by either (a) snowball sampling via online gaming forums, or (b) via a bespoke Facebook group. Once the participants had accessed the study's home page, they were presented with the statements. These were presented to participants online using customized QM software (Flash $Q)$. This includes physical "drag-and-drop" sorting of each statement, similar to sorting paper statements on an offline tabletop, and is available as an open-source demo from http://www.hackert.biz/flashq/home. Each participant was shown all 56 statements in a random order and asked to "drag and drop" these into one of three virtual piles (i.e., a pile of statements that participants agreed with, a pile of statements that participants disagreed with, and a neutral pile where participants neither agreed nor disagreed). On completion, participants were then presented with a Q-pyramid. Here, participants were asked to review each of their three piles and to place the statements within the corresponding areas on the pyramid. Statements that they disagreed with were placed between -6 and -2 ; neutral statements were placed between -1 and +1 ; statements they agreed with were placed within +2 and +6 . The more agreeable or disagreeable the statement, the closer it was placed to the pyramid's pole. Participants were then asked to give a written explanation for their choice of the sorted $(-6$ and +6$)$ statements. Finally, participants were asked to provide demographic information (i.e., age, gender), details of their gaming habits, personal reasons for gaming, and so on.

\section{Results}

Participant Q-sorts were analyzed using $P C Q$ for Windows. This program allows for the easy extraction of centroid factors and their rotation. Six factors were elicited and then subject to varimax rotation analyses (200 iterations). The six factors accounted for 31 of the original 40 sorts. Of the unaccounted for factors, three were found to be confounded and six nonsignificant. The six factors (Factors A-F) are described in more detail below. All statements are followed by statement number and sort position. Singular positive or negative numbers relate to the sorting position of an already highlighted statement.

\section{Factor $A$ (story-driven solo gamer)}

Gamers loading upon Factor A $(n=7)$ played computer games for a mean average of 17 hours per week. These gamers played alone for personal fulfillment, as shown by the high scoring of statement 6 ("I game for personal enjoyment, not to be better than others"; +6$)$, and by the fact that these gamers do not see it as important to compare achievements with others, as highlighted by statement 5 ("It is important for me to be top of a leader board within a game"; -5$)$. These gamers were motivated because they "play games because of their immersive background story" (statement $23,+6$ ). These gamers were motivated by the holistic nature of a wellwritten story-based game. They were motivated in finishing side missions, and never just "play to finish a level" (statement $30,-6)$. They were also driven to play games with good audio-visuals (statement $41,+5$ ). Their motivation to continue through a game's immersive story made them believe that "leveling up" (i.e., gaining enough points or abilities in order to move a player's character onto the next level) was something that had to be actively achieved (statement 28, $-5)$. These gamers regularly lost track of time when playing (statement $39,+5)$. The most preferred types of games played were the new high-definition graphical, story-driven, singleplayer games.

\section{Factor B (social gamer)}

Gamers loading upon Factor B $(n=5)$ played computer games for a mean average of 12 hours per week. These gamers primarily viewed their gaming as a social activity. This was highlighted by the very negative attitudes toward statement 4 ("I prefer playing alone, or playing single-player games"; -5) and statement 56 ("I choose to game alone so I can concentrate fully"; -5$)$. These gamers were not motivated by the external achievements offered by some console manufacturers (statement $33,-6$ ). However, these gamers were motivated by and were loyal to certain established franchises (statement $45,+5$ ), and experienced excitement at hearing certain in-game music (statement $44,+5$ ). These gamers did not set strict time limits upon their gaming activities (statement $40,-6)$, leading to regularly losing track of time while playing (statement $39,+6$ ). Saving their game data regularly was very important to these gamers (statement $11,+6$ ). Furthermore, they regularly noticed in-game advertisements (statement $52,-5$ ). Games played by these gamers were either social multiplayer and/or placed emphasis on social gaming.

\section{Factor $C$ (solo limited gamer)}

Gamers loading upon Factor C $(n=6)$ played computer games for a mean average of 13 hours per week. These 
gamers did not play to meet new people (statement $1,-5$ ) or as a social activity with friends (statement $55,-5)$. These gamers preferred to play single-player games (statement 4 , +5 ; statement $54,+6$ ). These gamers did not compare their achievements with other gamers as illustrated by the negative attitude toward statement 5 ("It is important for me to be top of a leader board within a game"; -6$)$. These gamers played games they liked the look of and not because they contained a particular character or held an endorsement/license (statement $46,+5$ : statement $49,-5$ : statement $50,+6$ ). They regularly used any data-saving facility offered within the game (statement 11, +5), and did not choose to create their own character or avatar (statement 19, -6). These gamers played a wide range of games, but played them more casually than other types of gamer. These gamers knew what they wanted from a game and preferred instant gratification.

\section{Factor $D$ (hardcore online gamer)}

Gamers loading upon Factor D $(n=3)$ played computer games for a mean average of 18 hours per week and did not put restraints upon their gaming (statement 40,-5). These gamers considered being part of a social group as a very important motivation in playing a particular game (statement $3,+5)$. They placed importance upon external rewards such as Xbox LIVE achievements and PSN trophies (statement 34, $-5)$. Although they played a wide range of different genres (statement $25,-6)$, they were very loyal to particular franchises within these genres (statement $45,+5$ ). These gamers also placed high importance upon the graphics of a game (statement 42,-5), and "cut scenes" were viewed as experience enhancing (statement $18,+5$ ). They regularly lost track of time while gaming (statement $39,+6$ ), and, if interrupted, they returned to the game as quickly as possible (statement $16,-6)$. Games played by these gamers were all individual titles from within wider franchises. Many of the games they preferred to play had very distinctive soundtracks or were music-based games (e.g., Guitar Hero).

\section{Factor $E$ (control/identity solo gamer)}

Gamers loading upon Factor E $(n=6)$ played computer games for a mean average of 18 hours per week. These gamers were motivated to play games for personal enjoyment rather than to compete with others (statement $6,+6$ ). They were motivated to play games that incorporated a strong storyline (statement 24,-6), and forwent the latest highdefinition graphics in order to experience quality game play (statement $42,+5$ ). They were not motivated by game violence (statement $48,+5$ ) and did not set time limits upon their gaming (statement $40,-5$ ). They viewed "cut scenes" as an important part of developing a strong and coherent storyline. This was illustrated by their very negative attitude toward statement 17 ("It annoys me and breaks up my enjoyment of a game when I have to sit through cut scenes or loading screens"; -5). Although these gamers played a wide range of games (statement $26,+6$ ), they also completed all side missions (statement $30,-6$ ). These gamers were highly motivated by creating their own character or avatar within a game. This was illustrated by their negative attitude toward statement 20 ("I generally play as a standard or predefined character"; -5$)$. They played a diverse range of games, par- ticularly story-driven games that allowed for elements of character choice or development. The story-driven game examples mostly came from the role-playing game (RPG) genre that allowed for the completion of many world-expanding side missions.

\section{Factor $F$ (casual gamer)}

Gamers loading upon Factor F $(n=4)$ played computer games for a mean average of 8 hours per week. These gamers were not motivated to play as a part of a social group (statement $3,-6)$ or by the need to top a leader board (statement $5,-5)$, but played for their own enjoyment (statement $6,+6)$. These gamers did not seek external help, choosing instead to work out their problems alone (statement $8,+5$ ), and they did not return to a game quickly when interrupted (statement $16,+5$ ). They played only when they had the time to do so, and when this was the case, they did not worry about restricting their gaming time (statement 40,-6). They placed a lot of emphasis upon game graphics. These gamers took every game at its own merits, choosing to play a game that looked good rather than being attached to a film or incorporating a character (statement $46,+5$ ). As a result of this, they were unlikely to have played all the games within a franchise (statement $45,-5$ ). Because of the casual nature of their gaming, they played graphically good, short games or mission-based games in order to fit a quick game into their lives.

\section{Discussion}

Findings from this study revealed a number of separate factors that appear to differentiate types of gamer. Each factor identified comprised a set of motivations that a gamer had for choosing to play particular games. Factor A gamers played games as a very personal experience. They were driven by the internal story, graphics, and sound of a game rather than by a sense of social comparison or competition with other gamers. Furthermore, they were motivated by holistic achievement within the game, and in order to do this, they complete all side missions. They were motivated by the rewards offered by "leveling up" a character, but regularly lost track of time when playing. Factor B gamers were motivated to play games for much more social reasons. However, they did not compare achievements on online gaming leader boards. These gamers were also motivated by sound within a game, although the graphics were not a major source of their gaming motivation. Because these players viewed gaming as a social pastime, they did not actively set time limits. They still experienced time loss, but did not view this as anything negative. The motivational characteristics of Factor B gamers were completely different from Factor A gamers (i.e., social rather than personal escape). However, both types were motivated by the sound elements of a game, and stated this as important.

Factor $C$ gamers were motivated by similar things to Factor A gamers in that they were motivated to play for personal experience rather than for a social experience. They were motivated by internal factors rather than external factors such as the social comparison offered by a gaming leader board. These gamers, like Factor A gamers, were motivated by the graphics of a game. However, unlike Factor A and B gamers, they did not place any importance on in-game sound. These 
gamers differed from Factor A gamers in terms of their strict personal limits on time spent gaming. These time limits meant that Factor $C$ gamers did not experience time loss. This, along with their lack of any identification for the in-game characters, suggests that Factor $C$ gamers were not motivated to play because of any immersive or escapist properties of the game. Factor D gamers were motivated to play for similar social reasons to Factor B gamers. However, Factor D gamers were motivated by social interaction with other online gamers (whereas Factor B gamers were not). Possibly as a result of online social interaction, Factor D gamers chose to compare their achievements to those of others through gaming leader boards. These gamers were motivated by the look of the game offered by high-quality graphics. The social and graphical importance of games to these players suggests they were regularly reminded of a game by external stimuli within their non-gaming lives. Factor D gamers highlighted losing track of time when playing games, and stated that they did not choose time limits for their gaming. If interrupted, they were motivated to recommence gaming at the next earliest opportunity.

Factor E gamers were the only type of gamer not to find the graphical or sound quality of a game important. These gamers were motivated more by good game play than by high-quality graphics. They saw cut-scene movies as very important in establishing a coherent and immersive story line. They identified greatly with any personally constructed or game-given avatar or character. Because of this character identification (coupled with the fact they chose to play games with coherent stories), Factor E gamers attempted to complete the entire game. When they were stuck, they sought help from external sources. Factor F gamers were identified as the most casual gamers. They played for the least amount of time and stated that they were not motivated by loyalty to a brand or franchise (choosing instead to play games that they thought looked good). These choices were not based upon external sources, and included many games from different genres. They did not experience time loss and did not appear to suffer time loss while playing games.

Although the analyses uncovered six distinct type of gamers based on preference for gaming structural characteristics, the different types incorporated some similar elements that in some cases appeared to motivate gamers and in other cases did not. The similar elements were: personal and/ or social motivation for gaming; experiencing subjective time loss; and the importance of high-quality sound and graphics. A major feedback mechanism for social competition in video games is the player leader board that can be found both offline (e.g., "Hall of Fame" screen on arcade video games) and online (e.g., multiplayer leader boards).

Despite prior research suggesting that individuals are highly motivated by social competition (facilitated by the comparison of achievements), ${ }^{7}$ the findings from this study appear to question this. The only type of gamer where players chose to compare achievements upon a leader board was the Factor D gamer. Only when viewing games as a social action do gamers compare themselves with others, either online (Factor D) or offline (Factor B). However, this does not appear to be at all important for other types of gamers. Other types of gamer identified here view gaming as a personal experience, motivated by their desire to complete the game for personal gratification (Factor A, C, and E gamers) or by the casual view of games (Factor F gamers). These findings question the importance of social competition as a motivational component within modern computer games. The results suggest that this factor is only important to a small highly specific group of gamers (Factor B and D gamers). The findings suggest that gamers within the current study were more likely to be motivated by elements of a game that encouraged a feeling of personal accomplishment and achievement. This is congruent with the "challenge" motivation. ${ }^{8}$ These structural characteristics would be high-quality graphics and sound, well-written characters, detailed stories, and well-constructed cut-scenes.

Factor A, B, and D gamers all highlighted that they "lost time" when playing. However, these gamers were affected differently by subjective time loss. Wood et al. ${ }^{9}$ studied gamers' subjective experiences of time loss and reported that gamers may experience either positive or negative manifestations of time loss. They suggested that time loss may be an indication of entering fully into the fantasy world. This was not seen as necessarily bad especially for gamers not confining their playing to personal time limits (Factor B gamers). These gamers played when they had time that they did not mind losing. This leads to the view of time loss as an enjoyable experiential aspect of gaming. The time loss experienced by Factor $\mathrm{A}$ and $\mathrm{D}$ gamers is illustrative of the immersive experience they sought from gaming such as the story-driven personal accomplishment (Factor A) or social interaction and competition (Factor D). Further research is needed to understand the effects that time loss has upon each gamer type, be this positive or negative. Further research is also needed to understand if the time loss is a motivational aspect of gaming or a consequence of playing because of other motivational structures, such as a well-constructed story, high-quality graphics, and/or the maintenance of social relationships.

Gaming scholars have argued that the graphics and sound effects in games enable a more realistic and immersive context for the video game's reward and storytelling design. ${ }^{4}$ This argument is certainly corroborated by the present study. All gamers (apart from Factor E) talk of the necessity for good graphics and even more importantly high-quality sound. There is also the question of gamers being motivated by different structural features depending upon the amount of time per week they choose to spend gaming. The results presented here suggest that motivations may not change depending upon the duration of time spent gaming, and that individuals are continually motivated by similar structures within games. However, this hypothesis would need to be tested within a longitudinal study comparing a number of Q-sorts completed by the same individual over an extended period.

Although the current study highlights the use of Qmethodology as a relevant and applicable method, it clearly has limitations. The study had a sample of only 40 gamers. Although this is within the parameters for QM outlined by Watts and Stenner, ${ }^{6}$ it is still a relatively small sample. Further studies are needed to validate and assess the accuracy of the structural factors resulting from this study. The study also utilized self-report data that may have led to socially desirable responses by the gamers. The study highlights to researchers and the gaming industry that gamers are not all motivated by the same aspects of games. It also highlights the importance of graphics and sound to most gamers, as well as the importance of strong storytelling devices. Future research is needed in the form of in-depth qualitative interviews dis- 


\section{VIDEO-GAME PLAY MOTIVATION}

cussing each factor with the gamers. This will hopefully validate and offer further understanding of the present study.

\section{Disclosure Statement}

AU3 No competing financial interests exist.

\section{References}

1. Jansz J, Tanis M. Appeal of playing online first person shooter games. CyberPsychology \& Behavior 2007; 10:133-6.

2. Kim Y, Ross S. An exploration of motives in sporting video gaming. International Journal of Sports Marketing \& Sponsorship 2006. Available online: http://www.imrpublications .com/JSMS/index.cgi?type $=$ sumabs\&vol $=8 \&$ num $=1 \& p a p=$ $3 \&$ id $=149$ (accessed Oct. 23, 2009).

3. Wood RTA, Griffiths MD, Chappell D, et al. The structural characteristics of video games: A psycho-structural analysis. CyberPsychology \& Behavior 2004; 7:1-10.

4. King D, Delfabbro P, Griffiths MD. Video game structural characteristics: A new psychological taxonomy. International Journal of Mental Health \& Addiction 2009. DOI: 10.1007/s11469-009-9206-4.

5. Cross RM. Exploring attitudes: The case for Q-methodology. Health Education Research Theory \& Practice 2005; 20:20613.

6. Watts S, Stenner P. Doing Q methodology: Theory, method and interpretation. Qualitative Research in Psychology 2005; 2:67-91.
7. Vorderer P, Hartmann T, Klimmt C. (2003) Explaining the enjoyment of playing video games: The role of competition. In Marinelli P, ed. Proceedings of the Second International Conference on Entertainment Computing (ICEC 2003). Pittsburgh, NY: ACM, pp. 1-8.

8. Sherry J, Lucas K, Greenberg B, et al. (2004) Video game uses and gratifications as predictors of use and game preference. In Vorderer P, Jennings B, eds. Playing video games: Motives responses and consequences. Mahwah, NJ: Lawrence Erlbaum Associates, pp. 213-24.

9. Wood RTA, Griffiths MD, Parke A. Experiences of time loss among videogame players: An empirical study. CyberPsychology \& Behavior 2007; 10:45-56.

10. Salen K, Zimmerman E. (2004) Rules of play: Game design fundamentals. Cambridge: MIT Press.

Address correspondence to: Dr. Mark Griffiths International Gaming Research Unit Psychology Division Nottingham Trent University Burton Street Nottingham NG1 $4 B U$ $U K$

E-mail: mark.griffiths@ntu.ac.uk 
CYBER-2009-0361-Westwood_1P.3D 02/22/10 12:15pm Page 6 


\section{AUTHOR QUERY FOR CYBER-2009-0361-WESTWOOD_1P}

AU1: Please check context of this sentence. It does not appear to fit in with the surrounding content. AU2: No text citation for reference 10. Please add.

AU3: Please verify disclosure statement is correct. If not, please revise. 